University of Nebraska - Lincoln

DigitalCommons@University of Nebraska - Lincoln

Norman R. Simon Papers

Research Papers in Physics and Astronomy

8-1969

\title{
An Explanation For The Blue Sequence Of Variable Stars
}

Richard Stothers

Goddard Institute for Space Studies

Norman R. Simon

University of Nebraska - Lincoln, nsimon@unl.edu

Follow this and additional works at: https://digitalcommons.unl.edu/physicssimon

Stothers, Richard and Simon, Norman R., "An Explanation For The Blue Sequence Of Variable Stars" (1969). Norman R. Simon Papers. 25.

https://digitalcommons.unl.edu/physicssimon/25

This Article is brought to you for free and open access by the Research Papers in Physics and Astronomy at DigitalCommons@University of Nebraska - Lincoln. It has been accepted for inclusion in Norman R. Simon Papers by an authorized administrator of DigitalCommons@University of Nebraska - Lincoln. 
The Astrophysical Journal, Vol. 157, August 1969

(C) 1969. The University of Chicago. All rights reserved. Printed in U.S.A.

\title{
AN EXPLANATION FOR THE BLUE SEQUENCE OF VARIABLE STARS
}

\author{
RICHARD STOTHERS AND NORMAN R. SIMON \\ Goddard Institute for Space Studies, NASA, New York \\ Received November 29, 1968; revised February 7, 1969
}

\begin{abstract}
Nuclear energy of the stellar core is found to be the direct source of energy fed into radial pulsations in two classes of blue variable stars. The $\mu$-mechanism (reversal of the gradient of mean molecular weight, which reduces the central condensation) or the $\beta$-mechanism (high radiation pressure) can explain the basic variability of $\beta$ Cephei stars if they are burning hydrogen in their cores, have accreted a heliumrich envelope, and are the luminous secondaries of close binary systems. If the composition reversal does not lead to significant mixing in the envelope, the $\mu$-mechanism will operate in inhomogeneous stars of 6-95 $M \odot$. If rapid mixing takes place, the $\beta$-mechanism will operate in completely homogeneous stars of $12-60 M \odot$. The $\beta$-mechanism can also explain the variability of many Wolf-Rayet stars if they are burning helium in their cores, have masses in excess of $\sim 8 M \odot$ with a thin or absent hydrogen envelope, and are the luminous primaries of close binary systems.
\end{abstract}

\section{INTRODUCTION}

One of the long-standing challenges to astrophysical theory has been the energizing mechanism which sustains the instability of blue variable stars. Neither the $\beta$ Cephei stars nor the irregular variables have been successfully explained by conventional theories of stellar evolution and pulsation (Eddington 1926; Ledoux 1941, 1951; Zhevakin 1953, 1959; Cox 1955; Cox and Whitney 1958; Schwarzschild and Härm 1959; Gurm 1963; Christy 1966; Underhill 1966; Stothers and Simon 1968). Recently, however, at least some of the Wolf-Rayet stars may have been properly interpreted in terms of the $\beta$-mechanism (Simon 1967; Simon and Stothers 1969a). But neither the $\beta$-mechanism nor the $\kappa$-mechanism has seemed to work for the other variables, since the central condensation was found to be too high and too low, respectively, for the nuclear-energy sources or the zones of hydrogen and helium ionization to interact with the pulsations. Underhill (1966) has suggested that the region of ultimate ionization of the CNO elements might provide an adequate site for the operation of the $\kappa$-mechanism in $\beta$ Cephei stars. The $\kappa$-mechanism can be ruled out, however, by the following comparison with stars in the classical Cepheid strip, where the $\kappa$-mechanism is known to operate in the zones of hydrogen and helium ionization: (1) the abundance of the ionizing element group is only a few percent by mass, (2) the period-spectrum relation has a negative slope, (3) the phase lag between the light curve and the radial-velocity curve is $90^{\circ}$ instead of $180^{\circ},(4)$ the light amplitude has an abnormal correlation with velocity amplitude, and (5) no $\beta$ Cephei strip would exist for any stage of ionization of the CNO elements besides the ultimate. With the exception of the aforementioned references, nearly all theories of the blue variables have so far been concerned chiefly with the surface manifestations of the phenomenon.

In this paper, we propose a new theory, termed here the $\mu$ mechanism, which accounts not only for the basic surface manifestations but also for the other circumstances surrounding these stars, especially their pulsational instability. A new application of the $\beta$-mechanism is also found for these stars.

\section{MU MECHANISM}

It is well known (e.g., Ledoux and Walraven 1958) that the fundamental eigenfrequency of radial pulsation in a star can be reduced either by increasing the radiation 
pressure ( $\beta$-mechanism) or by lowering the central condensation. Simon and Stothers $(1969 b)$ have shown that, if the eigenfrequency drops below a critical value $\left(\omega^{2} \sim 3\right)$, nuclear-energized pulsational instability develops (over a wide range of types of stellar configuration), provided only that the temperature exponent of the rate of nuclearenergy generation is high enough; hydrogen or helium burning fulfills this condition. While the $\beta$-mechanism does not work for normally evolving stars, we have discovered a general mechanism which does reduce the central condensation.

By adopting a reversal of the gradient of mean molecular weight $(\mu)$, it is possible to depress the central condensation of a star. The heavier material near the surface serves to compress the envelope while the central density remains virtually unaltered, being mainly determined by the nuclear-energy generation which must supply the (unaffected) luminosity.

It is here assumed that such a configuration arises as the result of mass exchange in a binary system. Several investigations of stellar evolution in massive binary systems have shown that the initially more massive component, evolving first, can lose most of its extended envelope to its unevolved companion on a fast time scale, followed by a phase of slower mass loss (Morton 1960; Kippenhahn and Weigert 1967; Paczynski 1967). Thus the companion accretes first a zero-age hydrogen-rich material and then, on top of that, a progressively helium-enriched material. It is this secondary star which we propose to study now.

\section{THEORETICAL MODELS}

Stothers (1965) seems to have been the only investigator to propose the main phase of hydrogen burning as the proper evolutionary state of $\beta$ Cephei stars. However, van Hoof (1965) has subsequently brought strong additional evidence to bear in support of this hypothesis. Therefore, we have decided to compute models with helium-rich surfaces for various stellar masses and evolutionary stages during central hydrogen burning and to test the models for stability against radial pulsations, in the linear quasi-adiabatic approximation.

The reversal of the gradient of mean molecular weight in the envelopes of these stars is not expected to cause a density inversion, but it might nevertheless produce an instability whereby mixing currents convect helium down toward the center (Spiegel 1969). The rate of mixing critically affects the distribution of hydrogen and helium throughout the star. Slow mixing will not destroy the $\mu$-gradient, because helium is continually being replenished at the surface through (1) the expansion of the secondary into the surrounding disk of material originating from the primary, (2) the normal gravitational infall of the disk material onto the surface of the secondary, and (3) the slowly continuing loss of matter from the primary even after it has become a helium star. On the other hand, fast mixing between surface and center will tend to keep the star chemically homogenized during evolution-accreted helium will be convected down toward the center, and central helium, produced by active hydrogen burning, will be convected up toward the surface. Since the rate of mixing is not known theoretically at the present time, we have considered two extreme cases: no mixing and complete mixing. The truth must lie somewhere between these two extremes.

\section{a) No Mixing}

The initial chemical composition of the interior region is assumed to be $X=0.70$, $Z=0.03$, and $X_{\mathrm{CNO}}=Z / 2$ (in the usual notation). For simplicity, we have replaced the gradient of mean molecular weight in the surface region by a discontinuity, thus putting all the excess helium in a single homogeneous zone near the surface. However, to satisfy formally the standard condition of convective neutrality, it is necessary in the models of higher mass to introduce a semiconvective zone of the type suggested by Schwarzschild and Härm (1958) between the surface zone and the interior. 
The following useful mass fractions are defined: $q_{a}$, discontinuity at the base of the homogeneous surface zone; $q_{f}$, boundary of the convective core; and $q_{0}$, boundary of the helium-rich region left behind by the retreating convective core in the evolved models. A quantity $\lambda=d \log \mu / d \log q$ represents the composition gradient between $q_{0}$ and $q_{f}$. Our choice of $q_{0}$ has been somewhat arbitrary, because the previous history of the rate and amount of mass accretion for the evolved models is not known. We have adopted essentially the same expressions for the gas characteristics of the models as Morris and Demarque (1966) used for their models of the upper main sequence:

$$
P=P_{\mathrm{gas}} / \beta=k \rho T / \mu \mathrm{H}+a T^{4} / 3 ; \quad \epsilon=10^{-106}{ }^{6} X X X_{\mathrm{CNO} \rho} T^{15} ; \quad \kappa=\kappa_{s}+\kappa_{a} ;
$$

with

$$
\kappa_{s}=0.19(1+X), \quad \kappa_{a}=10^{23.2} Z[(1+X) \rho]^{067} T^{-321} .
$$

The net gain or loss rate of pulsational energy over a cycle is denoted by $L^{*}{ }_{P}$ (where the possible damping due to surface running waves is neglected). Because of the rapid change of $L_{P}^{*}$ with a small change in $q_{a}$ for any model, the pulsational stability or instability is very easy to determine.

For illustrative purposes, we shall present for each mass and central hydrogen content those particular models containing a helium zone at the surface which is sufficiently deep to provide a marginal pulsational instability (since this is probably most realistic physically). The most favorable arrangement for pulsational instability is a homogeneous surface zone with zero hydrogen content. Table 1 contains models with (1) $X_{R}=0$ and (2) the maximum $X_{R}$ for which the star is still marginally unstable.

In no case presented is the amount of mass in the helium zone at the surface incompatible with physical feasibility. In this sense, the largest feasible surface zone for a star of given mass (below $35 M \odot$ ) is determined by (1) taking a normally evolved star with a mass outside the convective core at hydrogen exhaustion equal to the required mass and (2) "inverting" this mass onto a secondary star of initially negligible mass. Above $35 M \odot$, the largest feasible surface zone is determined by "inverting" the envelope of

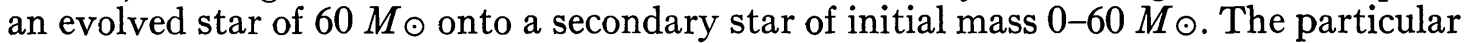
value of $60 M \odot$ refers to the maximum stable mass on the normal zero-age main sequence (Schwarzschild and Härm 1969; Stothers and Simon 1968). Table 2 presents the maximum permissible amount of excess helium for a number of stellar masses and the corresponding mass fraction that encompasses a homogeneous surface zone with $X_{R}=0$. According to our prescription, the highest possible mass for a secondary on the zero-age main sequence is $95 M \odot$, while the lowest unstable mass is only $6 M \odot$.

Figure 1 shows the theoretical results in a period- $M_{v}$ diagram. To convert the theoretical luminosities, we have adopted bolometric corrections due to Mihalas (1965) for main-sequence stars; the bolometric corrections are very insensitive to helium abundance and close to those for a perfect blackbody (Kuiper 1938). The trajectories in Figure 1 represent evolution off the zero-age main sequence (from left to right in the figure). The difference between models with differing surface helium content is virtually indiscernible and is therefore not shown. The mass is constant along each trajectory; however, the mass fraction contained in the helium zone at the surface increases both with decreasing stellar mass and with advancing evolution for models having the same $X_{R}$. Because of the rise of central condensation during normal stellar evolution, the necessary amount of surface helium to counteract this effect is larger for the more evolved models. The higher masses could not be calculated to completion of central hydrogen burning because of the eventual outbreak of widespread convection in the envelope. On the other hand, the lower masses became stable long before the end of hydrogen burning, because of the limitation imposed by the maximum permissible amount of accreted helium. 
TABLE 1

SeLECTED MODELS FOR $\boldsymbol{\beta}$ CEPHeI STARS

\begin{tabular}{|c|c|c|c|c|c|c|c|c|c|c|c|c|c|c|c|}
\hline \multirow{2}{*}{ Parameter } & \multicolumn{13}{|c|}{ INHOMOGENEOUS $(M / M \odot)$} & \multicolumn{2}{|c|}{ HOMOGENEOUS $\dagger(M / M \odot)$} \\
\hline & 5.9 & 12 & & & 15 & & & & & 30 & & 60 & 100 & 15 & 30 \\
\hline 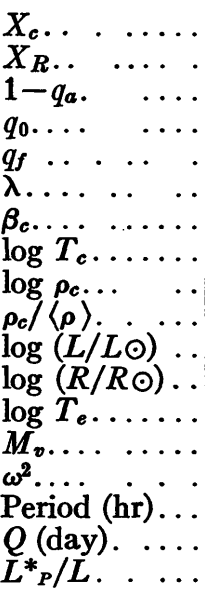 & 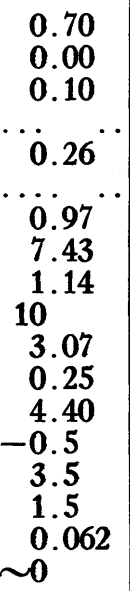 & $\begin{array}{c}0.20 \\
0.00 \\
0.15 \\
0.36 \\
0.23 \\
1.14 \\
0.83 \\
7.55 \\
0.85 \\
22 \\
4.36 \\
0.57 \\
4.57 \\
-2.9 \\
3.0 \\
3.4 \\
0.067 \\
\sim 0\end{array}$ & $\begin{array}{l}0.70 \\
0.00 \\
0.030 \\
\dot{0.40} \\
\dot{0} . \dot{ } .8 \\
7.51 \\
0.72 \\
12 \\
4.32 \\
0.57 \\
4.56 \\
-2.8 \\
3.3 \\
2.8 \\
0.064 \\
0\end{array}$ & $\begin{array}{c}0.70 \\
0.35 \\
0.34 \\
0.54 \\
\cdots \\
\cdots .87 \\
7.52 \\
0.69 \\
12 \\
4.46 \\
0.58 \\
4.59 \\
-3.0 \\
4.0 \\
2.6 \\
0.058 \\
0\end{array}$ & $\begin{array}{c}0.31 \\
0.00 \\
0.10 \\
0.40 \\
0.29 \\
1.09 \\
0.81 \\
7.55 \\
0.73 \\
20 \\
4.56 \\
0.63 \\
4.59 \\
-3.2 \\
2.9 \\
3.7 \\
0.068 \\
0\end{array}$ & $\begin{array}{c}0.34 \\
0.20 \\
0.25 \\
0.40 \\
0.29 \\
1.03 \\
0.80 \\
7.55 \\
0.69 \\
18 \\
4.62 \\
0.63 \\
4.60 \\
-3.3 \\
3.5 \\
3.4 \\
0.062 \\
\sim 0\end{array}$ & $\begin{array}{c}0.12 \\
0.00 \\
0.17 \\
0.40 \\
0.25 \\
1.29 \\
0.75 \\
7.59 \\
0.79 \\
26 \\
4.70 \\
0.65 \\
4.61 \\
-3.4 \\
2.6 \\
4.1 \\
0.071 \\
\sim 0\end{array}$ & $\begin{array}{c}0.70 \\
0.00 \\
0.007 \\
\cdots \\
0.56 \\
\cdots 0.77 \\
7.56 \\
0.48 \\
15 \\
5.09 \\
0.77 \\
4.65 \\
-4.1 \\
2.9 \\
4.3 \\
0.068 \\
0\end{array}$ & $\begin{array}{c}0.70 \\
0.45 \\
0.20 \\
0.56 \\
0.56 \\
0.62 \\
0.76 \\
7.56 \\
0.45 \\
16 \\
5.14 \\
0.79 \\
4.65 \\
-4.2 \\
3.4 \\
4.3 \\
0.063 \\
\sim 0\end{array}$ & $\begin{array}{l}0.29 \\
0.00 \\
0.040 \\
0.56 \\
0.41 \\
1.20 \\
0.65 \\
7.60 \\
0.52 \\
29 \\
5.30 \\
0.86 \\
4.66 \\
-4.5 \\
2.6 \\
6.1 \\
0.071 \\
\sim 0\end{array}$ & $\begin{array}{c}0.33 \\
0.20 \\
0.10 \\
0.56 \\
0.42 \\
1.12 \\
0.66 \\
7.60 \\
0.50 \\
25 \\
5.30 \\
0.84 \\
4.67 \\
-4.5 \\
2.9 \\
5.5 \\
0.068 \\
\sim 0\end{array}$ & \begin{tabular}{|l|}
0.28 \\
0.00 \\
0.012 \\
0.76 \\
0.54 \\
1.16 \\
0.49 \\
7.63 \\
0.33 \\
41 \\
5.90 \\
1.07 \\
4.70 \\
-5.7 \\
2.5 \\
9.2 \\
0.072 \\
$\sim 0$
\end{tabular} & $\begin{array}{c}0.34 \\
0.00 \\
0.003 \\
0.85 \\
0.64 \\
1.14 \\
0.42 \\
7.64 \\
0.20 \\
37 \\
6.24 \\
1.17 \\
4.74 \\
-6.3 \\
2.1 \\
11.0 \\
0.079 \\
\sim 0\end{array}$ & 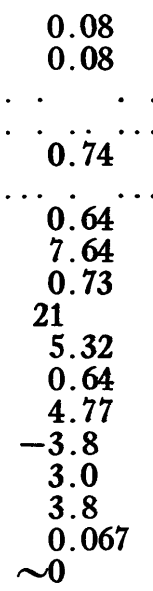 & $\begin{array}{c}0.36 \\
0.36 \\
\ldots \ldots \ldots \\
\ldots .74 \\
\cdots \ldots .64 \\
0.60 \\
7.60 \\
0.48 \\
21 \\
5.52 \\
0.82 \\
4.73 \\
-4.6 \\
3.0 \\
5.1 \\
0.067 \\
0\end{array}$ \\
\hline
\end{tabular}

* Calculated without surface running waves.

† Opacity taken as due only to electron scattering. Corresponding model for $63 M \odot$ may be found in Schwarzschild and Härm (1958, 1959). 


\section{b) Rapid Mixing}

In the event of a rapid mixing induced by mass accretion, the secondary will evolve with complete chemical homogeneity. When the growing stellar mass and mean molecular weight become such that the inequality $M / M \odot \geq 21 \mu^{-2}$ obtains, the star becomes pulsationally unstable because of the $\beta$-mechanism (Schwarzschild and Härm 1959). This inequality is only approximately true, because it is based on the assumption of a purely electron-scattering opacity. A constant opacity source, like electron scattering, exerts a relative destabilizing effect on the star. Nevertheless, the smallest

TABLE 2

MAXimum Amount of ACCRETEd Helium

\begin{tabular}{|c|c|c|c|c|c|}
\hline$M / M \odot$ & $\Delta Y d q$ & $\begin{array}{c}1-q_{a} \\
(Y=097)\end{array}$ & $M / M \odot$ & $\Delta Y d q$ & $\begin{array}{c}1-q_{a} \\
(Y=097)\end{array}$ \\
\hline $\begin{array}{r}4 \\
8 \\
8 \\
12 \\
15 \ldots\end{array}$ & $\begin{array}{ll}0 & 06 \\
0.08 \\
0.10 \\
0 & 12\end{array}$ & $\begin{array}{ll}0 & 08 \\
0 & 11 \\
0 & 15 \\
0 & 17\end{array}$ & $\begin{array}{l}20 \\
35 \\
60 \\
95\end{array}$ & $\begin{array}{ll}0 & 14 \\
0 & 20 \\
0 & 12 \\
0 & 07\end{array}$ & $\begin{array}{ll}0 & 20 \\
0 & 29 \\
0 & 17 \\
0 & 10\end{array}$ \\
\hline
\end{tabular}

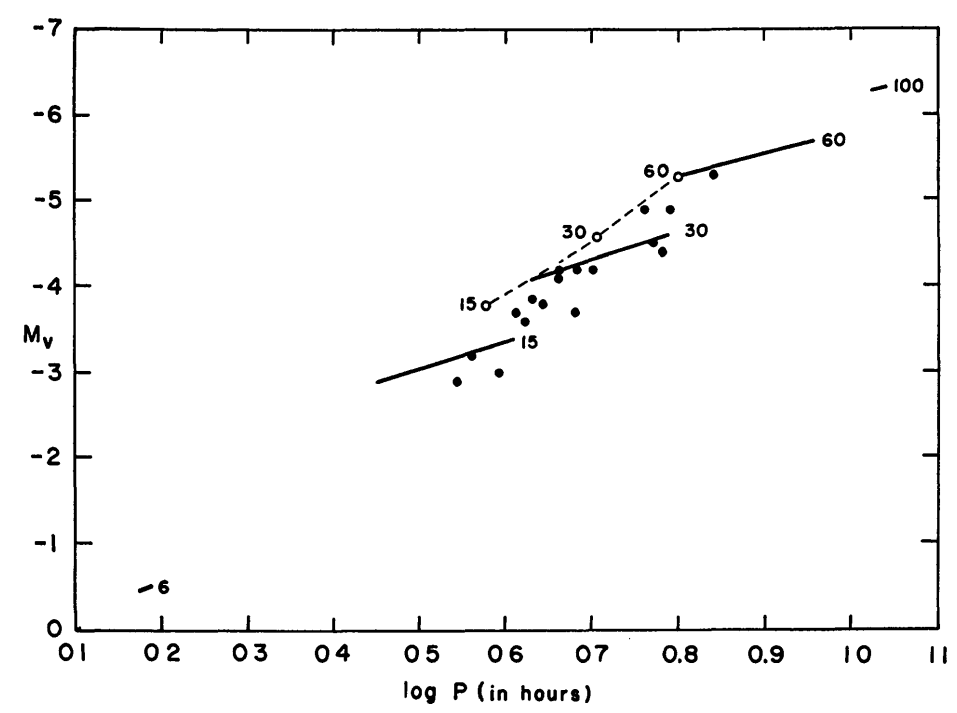

Fig. 1.-Period- $M_{v}$ diagram for $\beta$ Cephei stars and marginally unstable theoretical models during core-hydrogen burning. Coding as follows: observed stars (dots), inhomogeneous models based on $\mu$ mechanism (solid lines and heavy dashes), homogeneous models based on $\beta$-mechanism (open circles and light dashed line). Evolution in inhomogeneous models proceeds from left to right. For greater instability, model tracks should be shifted downward and to the left.

stellar mass which is physically possible on the hypothesis of complete mixing is likely to be about $12 M \odot$, a value which corresponds to $X \sim 0$.

Table 1 contains two marginally unstable models, calculated with a simple electronscattering opacity and with the assumption that the temperature gradient is radiative outside $q_{f}$ despite the mixing currents. Several such models are shown on Figure 1 as open circles; they define a locus of homogeneous stars obeying $(M / M \odot) \mu^{2}=21$. No inhomogeneous models appear for the following reason. Since mass accretion drives the mixing, the eventual cessation of the accretion permits development of an inhomogeneity between envelope and core, which stabilizes the star. 


\section{IV. $\beta$ CEPHEI STARS}

The known $\beta$ Cephei stars with adequate information are also plotted in Figure 1; the necessary data have been taken from Leung (1967). The agreement between our pulsationally unstable models and the observational data for $\beta$ Cephei stars is quite striking. Since our rather different kinds of models lie in the same region, the uncertainties in calculating them (particularly the distribution of helium) are probably unimportant for the basic comparison. Several conclusions, considerably different from the results of previous work, can be drawn from the comparison: (1) nuclear-energized radial pulsations explain the observed instability, (2) the masses of the stars range from $\sim 12 M \odot$ up close to the normal limit of stable masses, $\sim 60 M \odot$, and (3) all stages of evolution during core-hydrogen burning may be pulsationally unstable.

The critical question now is: Does the $\mu$-mechanism actually apply to the observed $\beta$ Cephei stars? In other words, is the binary hypothesis valid?

At least three $\beta$ Cephei stars are believed to be the brighter components of spectroscopic binary systems (Underhill 1966). It is also well known that all the $\beta$ Cephei stars have sharp lines and therefore very low rotational velocities (McNamara and Hansen 1961). This is unusual, since spectroscopically similar stars are normally characterized by very high rotational velocities. Low rotation, however, is always found in close binary systems. Struve (1950) and Ledoux (1951) had suggested earlier a binary hypothesis in a different interpretation.

The known orbital periods, 12-33 days (Underhill 1966), are certainly consistent with the assumption that the evolved primary has overflowed its Roche lobe. The subsequent formation of a very massive disk around the small secondary, leaving the primary star hydrogen-poor and undermassive, is a state which is actually observed in the $\beta$ Lyrae system (Woolf 1965). Since the secondary will not begin to pulsate, i.e., appear as a $\beta$ Cephei star, until its mass has grown enormously (the helium-rich portion of the disk being accreted last), the evolved primary ought to appear visually very faint because of its relatively low mass (even though it is a helium star) and its large bolometric correction (because helium stars are very hot). Thus, it is not surprising that companions of $\beta$ Cephei stars are detected in only a few cases.

In the theory invoking either the $\mu$ - or the $\beta$-mechanism, very little surface helium is necessary to destabilize a star of high mass. Therefore, the secondaries that are more massive are expected to become unstable far more easily and more quickly than the secondaries of lower mass. Consequently, a smaller proportion of $\beta$ Cephei stars (theoretically reaching zero at 6-12 $M \odot$ ) ought to be found at lower masses. On the other hand, stars of higher mass are, in general, numerically fewer in space (theoretically reaching zero at 60-95 $M \odot$ ). A maximum frequency may therefore be expected to occur between 15 and $30 M \odot$. The theoretical expectation is confirmed by the results of Figure 1 . Furthermore, the large proportion of early B giants which are $\beta$ Cephei stars can now be explained, because (1) $\beta$ Cephei stars need no longer be stars solely at the end of the phase of hydrogen burning in the core (see references in van Hoof 1965) and (2) the percentage of $B$ stars which are in binary systems is probably at least 50 percent (Petrie 1960). Finally, the high inferred masses of the $\beta$ Cephei stars are compatible with the presence of some of these evolved objects in associations where, in all known cases, the turnoff from the main sequence occurs between $\mathrm{O} 5$ and B0.

The assumption of rapid interior mixing in the $\beta$ Cephei stars accounts somewhat better for the observed mass cutoff at $\sim 12 M \odot$ and the narrowness of the observed sequence in the period- $M_{v}$ diagram. However, the assumption of slow interior mixing may be closer to the truth for the following reasons. First, the low-mass $\beta$ Cephei stars do not show a virtually hydrogenless spectrum. And, since the observational values 
of $M_{v}$ were derived from $\mathrm{H} \gamma$ equivalent widths calibrated in terms of normal $\mathrm{B}$ stars, and since these values agree well with values determined by cluster fitting (for the known association members), it is obvious that hydrogen cannot be grossly underabundant. For all masses in the observed range, the $\mu$-mechanism will work even with significant hydrogen abundance at the surface. It might be difficult with present model atmospheres to detect observationally the expected hydrogen deficiency with respect to the hydrogen abundance in normal stars (Underhill 1966). A second reason for suspecting little interior mixing is that, although this assumption predicts a mass cutoff near $6 M \odot$, such a prediction is merely formal-reflecting our adoption of a chemical discontinuity in the envelope instead of a true $\mu$-gradient and neglecting the effects of evolution in the core. Core evolution alone raises the mass cutoff to $12 M \odot$ (when $X_{c}=0.2$ ). Third, the sequence of completely mixed models in the period- $M_{v}$ diagram does not exactly coincide with the observed stellar sequence, and there is some scatter in the observed sequence which may not be due entirely to observational error in $M_{v}$. The unmixed models then give better coverage. Fourth and finally, the spectral types (B0.5-B2) and luminosity classes (III-IV) of the $\beta$ Cephei stars may be more appropriate for the unmixed models.

For either set of models, however, the predicted effective temperatures are hotter than those normally associated with early B stars. We can only suggest that a heliumrich composition in the atmosphere (Böhm-Vitense 1967) or a thick surrounding shell (see below) will make the spectral type appear later than normal for a very high temperature. A comparable discrepancy seems to exist between theoretical models and observed characteristics of the classical helium stars.

The large scatter in the "unreddened" colors of $\beta$ Cephei stars (Leung 1967) suggests (1) a spread in their evolutionary ages and (2) a small intrinsic reddening caused, for example, by a surrounding shell. The observed colors range from $(B-V)_{0}=-0.24$ to -0.28 , while our models and the temperature-color relation of Mihalas (1965) predict colors of -0.29 to -0.30 . The implied amount of intrinsic reddening is slight when compared with the observed amount for other kinds of blue variables, notably W-R stars (Smith 1968), P Cygni stars (Arkhipova 1963), and possibly HZ 29 (Stothers 1968). Furthermore, it is well known that shells of ejecta surround $\beta$ Cephei stars: for example, BW Vul (Odgers 1956) and $\sigma$ Sco (Morgan, Strömgren, and Johnson 1955). Such shells will arise in a natural way from self-regulation of the star's envelope. As the star accretes helium-rich material from the surrounding disk, the accreted helium tends (1) to reduce the stellar radius, because of the compression effect of its heavy weight, and (2) to increase the violence of the induced instability, leading to the ejection of shells of matter, i.e., of the recently accreted material. Indeed, good evidence exists for shock-wave phenomena in the atmospheres of $\beta$ Cephei stars (Struve 1955; Odgers 1956).

The basic light curve and radial-velocity curve of $\beta$ Cephei stars are sinusoidal. The phase lag between the light curve and the radial-velocity curve is $90^{\circ}$ (maximum light at minimum radius). Both characteristics are fully predicted by the linear quasi-adiabatic theory of nuclear-energized radial pulsations (e.g., Schwarzschild and Härm 1959), since the phase delay in energy generation by the CNO cycle is negligible (Cox 1955). The pulsation rise times are very short compared with the evolution times, so that steady pulsations will indeed be excited. The predicted ratio of light amplitude to radialvelocity amplitude is 2 or 3 times larger than actually observed. This slight discrepancy can be explained by the effect of a smaller opacity than we used near the surface, by progressive waves running off the photosphere which constitute a damping mechanism neglected in our analysis, and by the behavior of the bolometric correction (Lucy 1966), 
quite apart from our neglect of nonlinear and nonadiabatic effects. Despite these drawbacks, our models seem to be rather successful.

In this connection, we may remark that no excitation of radial-overtone pulsations was found in any of our models, even with the maximum allowable surface helium (cf. also Simon and Stothers 1969b). Thus the multiple-overtone hypothesis of van Hoof seems to be ruled out (van Hoof 1962; Stothers 1965). The problem remains of how to excite nonradial oscillations, as proposed in several theories which include rotation as a factor (Ledoux 1951, 1958; Chandrasekhar and Lebovitz 1962; Clement 1965). Because the pulsational energizing source lies very near the center, the damping time for nonradial oscillations should be less than the radial damping time of $10^{3}$ years. We also note that the envelope-averaged value of the generalized adiabatic exponent $\Gamma_{1}$, which is required to be a constant by the theory of Chandrasekhar and Lebovitz (where it should be approximately 1.6), is not a constant for our models, but ranges from 1.48 to 1.66. We have, therefore, no adequate explanation at present for the secondary periodicities which are observed.

\section{V. $\beta$-MECHANISM AND W-R STARS}

It was remarked earlier (Stothers 1965) that W-R stars form an "apparent extension" of the $\beta$ Cephei strip on the H-R diagram but that their instability is probably due to a different mechanism. Indeed, it now seems that many of the observed features of W-R stars can be interpreted on the basis of an unstable, hydrogen-poor primary star of a binary system (Paczynski 1967) and the $\beta$-mechanism (Simon 1967; Simon and Stothers $1969 a)$. The $\beta$-mechanism is found to work if the W-R stars are burning helium in their cores, have masses in excess of $\sim 8 M \odot$, and have sufficiently thin hydrogen envelopes. ${ }^{1}$ Observational evidence (summarized in the foregoing references) tends to support these rather severe conditions. Hence the W-R strip is parallel to, rather than an extension of, the $\beta$ Cephei strip. Actual W-R binary systems bear some resemblance to the forerunners of $\beta$ Cephei systems (cf. the massive disk in V444 Cyg [Underhill 1966]), but the OB secondaries are apparently stable stars and do not "drown out" the light of the primaries. On the contrary, the less massive primary of a W-R system is often the brighter star.

\section{CONCLUSION}

Our final picture of evolution in massive binary systems may be drawn as follows. If mass exchange is very large so that the secondary accretes a helium-rich envelope, the secondary may become a $\beta$ Cephei star because of the $\mu$ - or $\beta$-mechanism. If mass exchange is significantly less extensive or not yet completed (massive-disk stage) but is sufficient to rid the primary of its hydrogen envelope, the secondary is stable but the primary may become a luminous $W-R$ star because of the $\beta$-mechanism. In some systems, the exposed core of the primary may be sufficiently massive to become a W-R star, while at the same time the secondary evolves into a $\beta$ Cephei star. Superposed on the variations of the latter, short-period fluctuations with periods of less than 1 hour should occur; in fact, periodic phenomena of this sort have been observed in a few $\beta$ Cephei stars.

N. R. S. gratefully acknowledges the support of an NAS-NRC research associateship under the National Aeronautics and Space Administration. R. S. would like to thank Dr. E. A. Spiegel for a very helpful discussion.

${ }_{1}^{1}$ Paczynski (1967) independently suggested the $\beta$-mechanism in this context, on the basis of calculations by Boury and Ledoux (1965) for pure-helium stars, but made no pulsation calculations. The calculations of Simon and Stothers show that, for instability, the mass fraction contained in the hydrogen envelope must be $<0.05$ for $60 M \odot,<0.03$ for $15 M \odot$, and negligible for $8 M \odot$ (the last found by Boury and Ledoux). It should also be kept in mind that a few W-R stars may be single objects that lose their hydrogen envelopes through other mechanisms. 
No. 2, 1969

\section{REFERENCES}

Arkhipova, V. P. 1963, Astr. Zh., 40, 71.

Böhm-Vitense, E. 1967, $A$ p. J., 150, 483.

Boury, A., and Ledoux, P. 1965, Ann. d'ap., 28, 353.

Chandrasekhar, S., and Lebovitz, N. R. 1962, A p.J., 136, 1105.

Christy, R. F. 1966, Ap.J., 144, 108.

Clement, M. J. 1965, AP.J., 141, 1443.

Cox, J. P. 1955, Ap.J., 122, 286.

Cox, J. P., and Whitney, C. A. 1958, A p. J., 127, 561.

Eddington, A. S. 1926, The Internal Constitution of the Stars (Cambridge: Cambridge University Press).

Gurm, H. S. 1963, M.N.R.A.S., 126, 419.

Hoof, A. van 1962, Kleine Vëroff. d. Remeis-Sternwarte Bamberg, No. 34, p. 68. 1965, ibid., No. 40, p. 149.

Kippenhahn, R., and Weigert, A. 1967, Zs.f. Ap., 65, 251.

Kuiper, G. P. 1938, $A$ p. J., 88, 472.

Ledoux, P. 1941, Ap.J., 94, 537. . 1951, ibid., 114, 373

- 1958, ibid., 128, 336.

Ledoux, P., and Walraven, Th. 1958, Hdb. d. Phys., ed. S. Flügge (Berlin: Springer-Verlag), 51, 353.

Leung, K.-C. 1967, A p.J., 150, 223.

Lucy, L. B. 1966, $A . J ., 71,816$.

McNamara, D. H., and Hansen, K. 1961, A p. J., 134, 207.

Mihalas, D. 1965, Ap.J.Suppl., 9, 321.

Morgan, W. W., Strömgren, B., and Johnson, H. M. 1955, Ap. J., 121, 611.

Morris, S. C , and Demarque, P. 1966, Zs. f. Ap., 64, 238.

Morton, D. C. $1960, A$ p. J., 132, 146.

Odgers, G. J. 1956, Pub. Dom. Ap. Obs. Victoria, 10, 215.

Paczynski, B. 1967, Acta Astr., 17, 355.

Petrie, R. M. 1960, Ann. d'ap., 23, 744.

Schwarzschild, M., and Härm, R. 1958, A p.J., 128, 348. . 1959, ibid., 129, 637.

Simon, N. R. 1967, unpublished Ph.D. thesis, Yeshiva University.

Simon, N. R., and Stothers, R. 1969a, Ap. J., 155, 247. 1969b, ibid., 156, 377 .

Smith, L. F. 1968, M.N R.A.S., 140, 409.

Spiegel, E. A. 1969, Comments on Astrophysics and Space Physics 1, 57.

Stothers, R. 1965, A p.J., 141, 671 . . 1968, Ap.J (Letters), 154, L91.

Stothers, R., and Simon, N. R. 1968, Ap. J., 152, 233.

Struve, O. 1950, Ap.J., 112, 520.

. 1955, Pub. A.S.P., 67, 135.

Underhill, A. B. 1966, The Early Type Stars (Dordrecht: D. Reidel).

Woolf, N. J. 1965, Ap.J., 141, 155.

Zhevakin, S. A. 1953, Astr. Zh., 30, 161. 1959, ibid., 36, 996. 
